follow-up. Seizures considered generalized convulsive initially in 40 children, when captured by ictal EEG at 7 years of age, were of focal origin in 35 (87.5\%). Myoclonic, atypical absence, and photo- and pattern-sensitive seizures disappeared by 20 years of age in most typical cases. Patients with $<3$ episodes of convulsive status epilepticus in childhood and no EEG spikes on follow-up $(\mathrm{P}<0.001)$ had a seizure-free outcome as adults $(\mathrm{P}=0.018)$. Seizure freedom was not correlated with SCN1A mutation type, gender, type of DS, or generalized spike-waves on EEG. Of $26(83.9 \%)$ patients with persisting seizures as adults, $19(73 \%)$ had mostly nocturnal seizures; $10(38.5 \%)$ continued to have seizures provoked by fever, none with status epilepticus. At last follow-up, seizures were refractory in $84 \%$, and 5 patients $(16.1 \%)$ had $10-29$ seizures per month. Seven patients $(22.6 \%)$ spoke no words and $9(29 \%)$ spoke only several words. Five $(16.1 \%)$ made simple conversation and could read a little. Occipital alpha rhythms in follow-up EEGs at a mean age of 23.8 years correlated with less severe intellectual disability $(\mathrm{P}=0.002)$. Prevention of occurrence of convulsive status epilepticus in childhood was critically important for improved seizure outcome in DS in adulthood. (Akiyama M, Kobayashi K, Yoshinaga H, Ohtsuka Y. A long-term follow-up study of Dravet syndrome up to adulthood. Epilepsia June 2010;51:1043-1052). (Respond: Dr K Kobayashi, Department of Child Neurology, Okayama University Hospital, Shikatacho 2-chome 5-1, Kitaku, Okayama, Japan 700-8558. E-mail: k_koba@md.okayama-u.ac.jp).

COMMENT. In this long-term, follow-up study of patients diagnosed with Dravet syndrome, the main factor associated with improvement of seizure prognosis is the prevention of occurrence of convulsive status epilepticus in childhood. Details of treatment were not listed, except that the approval and introduction of topiramate in 2007 controlled seizures in 4 previously unresponsive patients.

\title{
EFFECTS OF DTP VACCINATION ON RAVET SYNDROME
}

An association between DTP vaccination and onset of seizures in 40 patients diagnosed with Dravet syndrome was investigated by retrospective analysis of medical and vaccination records at Heidelberg Hospital, Victoria, Australia. Patients with mutations in SCN1A whose first seizure was a convulsion were separated into 2 groups, those whose first seizure occurred on the day of or day after vaccination $(n=12)$ (the vaccination-proximate group), and those with seizure onset 2 days or more after vaccination $(n=25)$ or before vaccination $(n=3)$ (the vaccination-distant group). Mean age at seizure onset was 18.4 weeks (SD 5.9) in the vaccination-proximate group and 26.2 weeks (SD 8.1) in the vaccination-distant group (difference $\mathrm{p}=0.004$ ). Intellectual outcome, subsequent seizure type, and mutation type were similar in the two groups (p values >0.3). Intellectual outcome did not differ between patients who received vaccinations after seizure onset and those who did not. Vaccination might trigger earlier onset of seizures in children with SCN1A mutations and susceptibility to Dravet syndrome, but, by post-hoc analysis of data, vaccinations before or after onset of Dravet syndrome do not affect intellectual outcome. (McIntosh AM, McMahon J, Dibbens LM et al. Effects of vaccination on onset and outcome of Dravet syndrome: a retrospective study. Lancet Neurol June 2010;9:592-598). (Respond: Prof Samuel F Berkovic. E-mail: s.berkovic@unimelb.edu.au). 
COMMENT. Seizures in Dravet syndrome (DS) begin in the first year, usually at or around 6 months of age. Early seizures are typically prolonged and associated with fever or infection. Known seizure triggers include, in addition to fever and infection, hot water bath, and photic or pattern stimulation. Mutations in the SCN1A gene are found in $67 \%$ to $86 \%$ of DS cases. (Dravet C et al, 1982, 2005; Millichap JJ, Koh S, Laux LC, Nordli DR Jr. Dravet syndrome. When to suspect the diagnosis. Neurology 2009;73:1-1).

Wiznitzer M, in an editorial, (Lancet Neurol 2010;9:559-561) comments that the study by McIntosh and colleagues has limitations but it attempts to introduce science over speculation and bias in the question and controversy of pertussis vaccination and encephalopathy. Although 12 individuals with DS and a first seizure within 2 days of whole cell or acellular DTP had earlier onset than the 28 with a first seizure before or more distant from vaccination, their clinical outcome, including cognitive disability, was not different, and no worsening occurred in individuals who received further vaccinations. Both authors conclude that outcome is determined by the underlying disorder and not by proximity to vaccine administration.

\section{KETOGENIC DIET FOR EPILEPSY WITH TYPE 1 DIABETES}

Researchers at Medical University Vienna, Austria, report the efficacy and safety of the ketogenic diet (KD) in treatment of epilepsy in a 3-year 6 month-old girl with diabetes type 1 followed for 15 months. Right-sided spastic hemiparesis with widespread left hemisphere malformation were diagnosed at 9 months and were associated with focal tonic seizures during sleep. EEG-video monitoring showed interictal spikes in left central-parietal, and frontal and occipital electrodes. Seizure frequency (1-2 per week) increased at 30 months of age and because of side effects, the parents stopped antiepileptic drug treatment but agreed to a trial of the KD. John Hopkins' KD protocol was followed with a 2.5:1 ratio, and insulin requirement was reduced. Since the KD, no clinical seizures were reported but after 10 months, the EEG showed persistent epileptiform activity over the left hemisphere and 4 subclinical seizures, especially during sleep. Gains in language and motor skills were observed. No severe hypoglycemia or ketoacidosis occurred and metabolic control was maintained. The child refused ketogenic meals and the parents discontinued the diet after 15 months, and no clinically overt seizures were reported. (Dressler A, Reichofer E, Trimmel-Schwahofer P et al. Type 1 diabetes and epilepsy: efficacy and safety of the ketogenic diet. Epilepsia June 2010;51:1086-1089). (Respond: E-mail: anastasia.dressler@meduniwien.ac.at).

COMMENT. The authors conclude that diabetes does not preclude the use of the ketogenic diet in treatment of childhood epilepsy. Glycemic control is maintained without severe side effects, and development may be improved. 